\title{
Direct Explorations of Exoplanets with the Subaru Telescope and Beyond
}

\author{
Motohide Tamura ${ }^{1}$, Lyu Abe ${ }^{1}$ \\ and Subaru HiCIAO/SPICA coronagraph/JTPF teams \\ ${ }^{1}$ Exoplanet Project Office, National Astronomical Observatory, \\ Osawa 2-21-1, Mitaka, Tokyo 181-8588, Japan \\ email: hide@subaru.naoj.org
}

\begin{abstract}
We present a Japanese "roadmap" on direct extrasolar planet studies spanning from the current ground-based telescope to future IR/Opt space-based telescopes. Several searches for young planets and disks have been conducted with the Subaru $8.2 \mathrm{~m}$ telescope with adaptive optics (AO) infrared coronagraph, CIAO. The instrument will be soon upgraded to a new $\mathrm{AO}$ and a coronagraph with simultaneous spectral and polarimetric differential imaging modes (HiCIAO), which will significantly improve the contrast performance and hence the capability of young planet detection. A sensitive unbiased survey for extrasolar zodiacal emission around nearby stars will be conducted with the ASTRO-F space mission $(0.7 \mathrm{~m}$ telescope, $\sim 2-$ $200 \mu \mathrm{m})$ to be launched around the beginning of 2006. A successor space mission, SPICA (3.5m, 5-200 $\mu \mathrm{m}$ ), is also planned; its high sensitivity will enable the detection and characterization of outer-most planets around nearby stars, if any. For the studies of extrasolar terrestrial planets, a high contrast space telescope (HCST; $3.5 \mathrm{~m}, \sim 0.3-2 \mu \mathrm{m}$ ). We are also seeking for collaborations with or are considering to join to foreign missions. We describe an outline, status, and role of each project on the extrasolar planet studies.
\end{abstract}

Keywords. instrumentation: high angular resolution, planetary systems: formation, planetary systems: protoplanetary disks, stars: low-mass, brown dwarfs.

\section{A roadmap}

Figure 1 is a roadmap showing our approach to the explorations of exoplanets in Japan.

Our ground-based direct explorations are concentrated on the fully-operated and successful Subaru 8.2m telescope at Mauna Kea, Hawaii. Making use of the good observing site and the excellent image quality, the infrared coronagraph CIAO (Coronagraphic Imager with Adaptive Optics) has been used for various kinds of surveys, which is described in detail in the next section. Its description and scientific results are introduced in $\S 2$. Several indirect efforts are also sought on the Subaru telescope: an international collaboration on the N2K survey on the Doppler searches has been most productive (Sato et al. 2005). HiCIAO (Next generation High Contrast Imager with Adaptive Optics for the Subaru telescope) is the Subaru next generation coronagraph which is used with the new AO and its near-future upgrade to an extreme-AO system. This will be used for the more extensive surveys of young exoplanets, as described in $§ 3$. Regarding space-based explorations, the MIR and FIR space telescope, $A S T R O-F$, is developed by JAXA/ISAS and will be launched soon (Murakami 2000). This telescope has a similar size to the Spitzer telescope, but is optimized to all-sky survey observations. Although its low spatial resolution is not suitable for direct explorations, it will conduct an unbiased survey for faint disk emission. This enables us to make census of exo-zodi and disk evolution with a large sample of stars. As a next project in the OPT/IR community in Japan after Subaru/ASTRO-F, SPICA is currently heavily discussed, a cooled large single mirror 


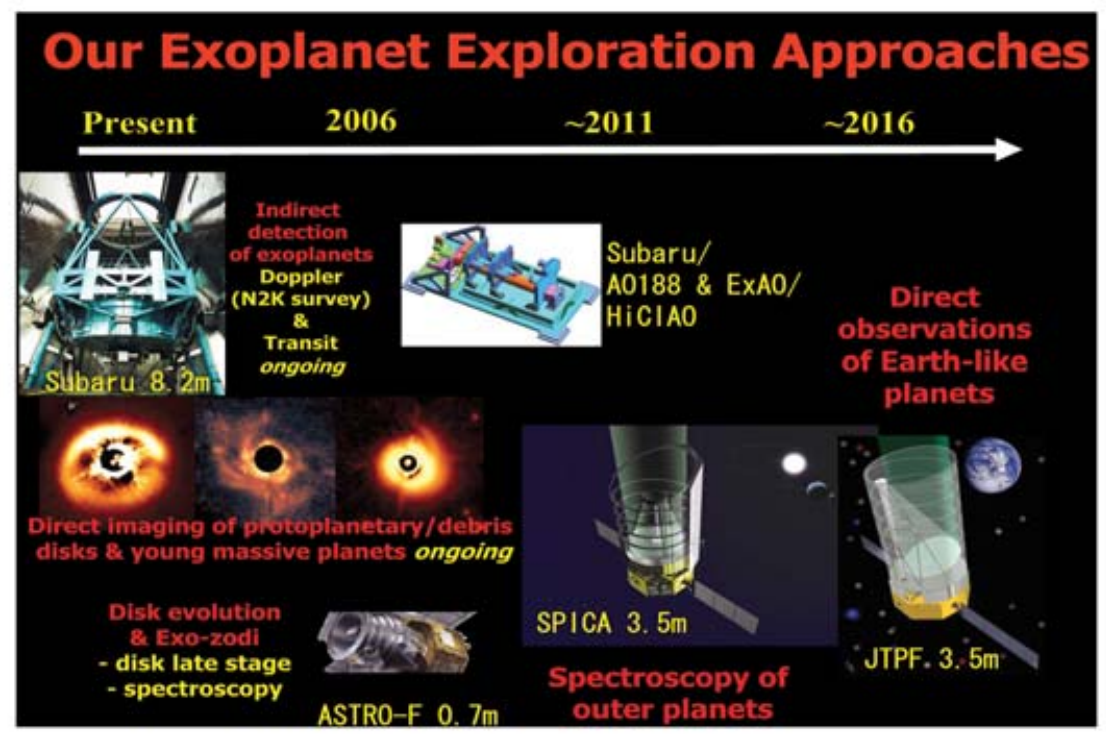

Figure 1. A roadmap of exoplanet explorations in Japan.

space-telescope. Because of its high sensitivity and acceptable resolution, the mission will serve for direct exoplanet explorations. Its coronagraph and exoplanet science are discussed in $\S 4$. A space-telescope dedicated for direct explorations of Earth-like planets is also under discussion in the OPT/IR community in Japan, as described in $§ 5$.

\section{Subaru CIAO - First dedicated infrared coronagraph on 8-10 m class telescopes}

Observationally, the direct imaging of both exoplanets and protoplanetary disks is extremely difficult: it requires a high sensitivity, a high resolution, and a high dynamic range or contrast at the same time. Such high sensitivity and high resolution are now simultaneously realized with the 8-10 meter class telescopes with adaptive optics (AO). The AO corrects for atmospheric perturbations in real-time, by the means of wavefront sensors and deformable mirrors. In the current Subaru AO system, 36-elements sensors and 36-elements mirrors are used. With the Subaru adaptive optics and infrared camera we regularly achieve a resolution of $0.07-0.10 \operatorname{arcsec}$ and a sensitivity of $22 \mathrm{mag}$ ( $5 \sigma$ in 1 hour) around $1.5-2 \mu \mathrm{m}$ ( $H$ and $K$ bands). However, a high contrast, that is, a capability to observe faint objects near bright objects, is another thing.

CIAO is the first among those equipped with a full cold coronagraph mode (with various cold occulting masks and Lyot stop optics) on the world 8-m class telescopes. Compared to previous coronagraphs, it has unique features of near-infrared operation and small occulting masks (down to 0.2 arcsec in diameter). See Tamura et al. (1998, 2000) for the instrument details.

CIAO has a comparable or higher contrast than other coronagraphs on the 8-m class telescopes (Table 1). See Nakajima et al. (2005) for a detailed performance discussion of CIAO. 
Table 1. Contrast comparison (from Nakajima et al. 2005)

\begin{tabular}{ccc}
\hline Telescope/Instrument & Contrast & Separation \\
\hline Subaru/CIAO & $\Delta \mathrm{K}=13$ & $\mathrm{r}>2.5^{\prime \prime}$ \\
Subaru/CIAO & $\Delta \mathrm{K}=11$ & $\mathrm{r}=1.2^{\prime \prime}$ \\
VLT/AO+Mask & $\Delta \mathrm{K}=13$ & $\mathrm{r}>3.0^{\prime \prime}$ \\
VLT/AO+Mask & $\Delta \mathrm{K}=10$ & $\mathrm{r}=1.0^{\prime \prime}$ \\
VLT/AO+SDI & $\Delta \mathrm{H}=9.5$ & $\mathrm{r}=0.5^{\prime \prime}$ \\
\hline
\end{tabular}

\subsection{CIAO disk searches}

CIAO has been successful to directly reveal the morphological diversity of protoplanetary disks, such as rings, spirals, and even others, with a high spatial resolution of $\sim 0.1^{\prime \prime}$. As shown in an inset of Figure 1, besides a well known ring-like disk around GG Tau, a clear spiral disk with several arms has been revealed around AB Aur, and a new-type of disk morphology, banana-split disk, has been recently discovered around HD 142527 (Fukagawa et al. 2004 and 2005). In addition, first $2-\mu$ m imaging polarimetry of $\beta$ Pic has demonstrated that the disk is composed of several planetesimal belts with a gap at $\mathrm{r} \sim 100$ AU (Tamura et al. 2005).

\subsection{CIAO companion searches}

Companion searches are greatly merited if the targets are younger; the younger companion is brighter and the contrast between the primary and the companion becomes lower (e.g., Burrows et al. 2001). This is one of the reasons why we explore YSO companions in nearby star forming regions.

DH Tau is a classical T Tauri star with an age of 0.1-4 Myr and a mass of 0.25-0.5 solar mass. CIAO coronagraphic images have revealed a companion of 15 mag at $H$ at $2.3^{\prime \prime}$ (330 AU) from the central star. This star is originally selected because it has a relatively large infrared polarization (Tamura \& Sato 1989), suggestive of the circumstellar structures. However, interestingly, no significant circumstellar disks or envelopes are detected except for the companion.

The companion shows the same proper motion of the Taurus cloud members, distinct from the background stars. Its near-infrared spectra show deep absorption band due to water and other metal features, all indicating low effective temperatures, but not as low as those of planetary mass objects $\left(\mathrm{T}_{\text {eff }} \sim 2700 \mathrm{~K}\right)$. With recent theoretical models, the companion is concluded as a companion young brown dwarf of about $40 \mathrm{M}_{J U P}$ (Itoh et al. 2005). This source is more or less similar to the recenly detected companion around GQ Lup and other handful companions around YSOs (Neuhauser et al. 2005; Liu 2005 in this proceedings).

Similar surveys are also nearby M stars using CIAO (Nakajima et al. 2005).

\section{HiCIAO - the Subaru next high contrast instrument}

HiCIAO is a new high-contrast instrument for the Subaru telescope, which is currently developed by NAOJ and University of Hawaii. NAOJ PI is M. Tamura and UH PI is K. Hodapp. HiCIAO will be used in conjunction with the new adaptive optics system (188 actuators and/or its laser guide star - AO188/LGSAO188) at the Infrared Nasmyth platform. It is designed as a flexible camera comprising several modules that can be configured into different modes of operation. The main modules are the AO module with its future extreme AO capability, the warm coronagraph module, the high contrast optics module, and the cold infrared camera module. HiCIAO will be the first instrument on 
the 8-m class telescopes which can combine coronagraphic techniques with simultaneous polarization and spectral differential imaging modes which minimizes the common path errors. The basic concept of such differential imaging is to split up the image into two or more images, and then use either different planes of polarization or different spectral filter bandpasses to produce a signal that distinguishes faint objects near a bright central object from scattered halo or residual speckles. This enables us to achieve a contrast improvement of at least 10 times better than before. The instrument specification is summarized in Table 2.

Table 2. HiCIAO overall specifications.

\begin{tabular}{|c|c|}
\hline \multirow{2}{*}{\multicolumn{2}{|c|}{$\begin{array}{l}\text { Platform } \quad \text { Subaru Nasmyth focus (IR) } \\
\text { Combined adaptive optics 188-elements curvature-sensing AO }\end{array}$}} \\
\hline & \\
\hline Wavelength coverage & $z J H K s$ (optimized at $\mathrm{H})$ \\
\hline Detector & $2048 \times 2048 \mathrm{HgCdTe}$ (Rockwell HAWAII-2RG) \\
\hline Detector controller & ASIC (Rockwell "Sidecar") \\
\hline Pixel scale & $0.01^{\prime \prime} /$ pixel (proper PSF sampling at J-band) \\
\hline Spatial resolution & $0.03^{\prime \prime}(J), 0.04^{\prime \prime}(H), 0.055^{\prime \prime}(K s)$ FWHM \\
\hline Strehl ratio & $0.6(J), 0.7(H), 0.8(K s) \mathrm{w} / 0.5^{\prime \prime}$ optical seeing \\
\hline Sensitivity & $H>23 \mathrm{mag}(5 \sigma, 1 \mathrm{hr})$ \\
\hline Contrast goal & $10^{4}$ at $0.1^{\prime \prime}, 10^{6}$ at $1^{\prime \prime}(\mathrm{w} / \mathrm{SDI}$ mode + coronagraph $)$ \\
\hline \multirow[t]{3}{*}{ DI mode } & Direct imaging \\
\hline & $20^{\prime \prime} \times 20^{\prime \prime} \mathrm{FOV}$, general purpose imaging \\
\hline & High throughput, w/wo coronagraph \\
\hline PDI mode & $\begin{array}{l}\text { Polarimetric (o-ray and e-ray) dual imaging (Wollaston prism) } \\
20^{\prime \prime} \times 10^{\prime \prime} \mathrm{FOV} \text {, polarized target imaging }\end{array}$ \\
\hline SDI mode & $\begin{array}{l}\text { Spectral simultaneous differential imaging (Double Wollaston prism) } \\
6^{\prime \prime} \times 6^{\prime \prime} \text { FOV, unpolarized target imaging }\end{array}$ \\
\hline \multicolumn{2}{|l|}{ Coronagraph* $^{*}$} \\
\hline Occulting masks & 3 sizes with real-time remote choice \\
\hline Lyot stops & Stop with spider/center-hole blocks \& rotation compensation \\
\hline
\end{tabular}

* Starting with the classical Lyot coronagraph. More sophisticated coronagraph will be implemented later.

The main sciences with Subaru/HiCIAO are summarized as follows.

- For directly detecting young (age $100 \mathrm{Myr}$ ) extrasolar planets and brown dwarfs, HiCIAO can be used to conduct a survey of $\sim 120$ nearby relatively young dwarfs. About 60 stars younger than $700 \mathrm{Myr}$ with spectral types between A and $\mathrm{K}$ are accessible from Subaru. These 60 stars and the $350 \mathrm{Myr}$ M dwarf sample ( 60 stars) will serve as the prime targets for the brown dwarf/planet. The inner radius of search will be reduced from $25 \mathrm{AU}$ to $10 \mathrm{AU}$ for a target at $10 \mathrm{pc}$ by the transition from CIAO to HiCIAO.

- For directly detecting very young (age 1-10 Myr) extrasolar planets and brown dwarfs, HiCIAO can be used to conduct a survey of $\sim 200 \mathrm{~T}$ Tauri stars in nearest star forming regions accessible from the northern hemisphere and to detect young Jupitermass companions down to an inner radius of $30 \mathrm{AU}$. With the availability of the laser guide star, the target can be extended to the optically faint sources including Class I YSOs.

- HiCIAO will be efficiently used to detect or set a limit on scattered light from disks around the young stars in nearby star forming regions. The spatial resolution is $0.03^{\prime \prime}$ (80 AU at the distance of the Taurus dark cloud) in the $J$-band, which is better than the 
HST resolution. The large sample of disk data will be used to trace the morphological evolution and diversity of YSO disks at the age of age $<1$ Myr to 10 Myr.

- HiCIAO will be extremely useful to detect or set a limit on scattered light from the known and new, large samples of Vega-like disks from the Spitzer and the ASTRO-F missions, which requires both high sensitivity and high contrast. The achievable spatial resolution is $0.6 \mathrm{AU}$ at the distance of $\beta$ Pic. The polarization differential imaging is crucial to study the small-scale and inner disk structures and dust grain properties. A synergy observation with the ASTRO-F will be considered.

- The targets for TPF are well pre-surveyed with HiCIAO for characterizing their circumstellar matter and detecting relatively "low"-contrast objects including background stars and galaxies.

An extensive survey dedicated to these sciences on the Subaru telescope will be a significant contribution to astronomy before the high contrast space telescope era. The application to other targets might include not only other galactic objects such as late-type stars but also extragalactic objects such as AGNs and quasars, especially with the laser guide stars, where the AO application has been relatively limited. A high throughput direct imaging will be useful for such objects.

\section{SPICA coronagraph}

The SPICA mission plans to launch a cooled $(4.5 \mathrm{~K})$ large $(3.5 \mathrm{~m})$ single-mirror telescope into a halo orbit around the Sun-Earth L2 orbit around the beginning of 2010s (Nakagawa 2000). Its unprecedented sensitivity at MIR (and FIR) and its simple telescope pupil make SPICA to be one of the best platforms to implement coronagraph instrument for exoplanet studies. The preliminary specification of the SPICA coronagraph is summarized in Table 3.

Table 3. SPICA coronagraph prelliminary specification.

\begin{tabular}{ll}
\hline Wavelength coverage & $5-27 \mu \mathrm{m}$ \\
Spectral resolution & $<200$ \\
Inner working distance & $3 \lambda / \mathrm{D}$ \\
Contrast goal & $10^{6}$ \\
Detector & $1 \mathrm{Kx} 1 \mathrm{~K}$ Si:AS IBC \\
Adaptive Optics & Cold Tip-Tilt mirror (to correct for the jitters) \\
& Cold Deformable Mirror (to correct for the mirror wavefront errors) \\
\hline
\end{tabular}

SPICA will target direct observations of self-luminous planets at $r>$ a few to $\sim 20 \mathrm{AU}$ of nearby $(<10 \mathrm{pc})$ stars. The detectable planets depend on their mass, age, and separation. If we assume the inner working distance of $3 \lambda / \mathrm{D}$, then at $\lambda=5 \mu \mathrm{m}$ we can detect $1 \mathrm{Gyr}, 2$ $\mathrm{M}_{J U P}$ planets at $\mathrm{r}>9 \mathrm{AU}$ around $10 \mathrm{pc}$ stars ( $\sim 30 \mathrm{G}-\mathrm{M}$ type stars). At $\lambda=20 \mu \mathrm{m}$ we can detect 5 Gyr, $2 \mathrm{M}_{J U P}$ planets at $\mathrm{r}>18 \mathrm{AU}$ around $10 \mathrm{pc}$ stars ( 150 G-M type stars). Very young planets and sub-brown dwarfs in nearby star forming regions and cold brown dwarfs are also good targets.

\section{JTPF}

JTPF (Japanese Terrestrial Planet Finder) is a future space mission whose main science driver is the extrasolar terrestrial planet studies. Th project is officially approved both by NAOJ and ISAS. In NAOJ, its activity is regarded as one of the main themes of the Phase A project which has started in April 2005. In JAXA/ISAS, the JTPF Working Group 
has been approved by the ISAS Science Steering Committee in 2002. Discussion has been made among both astronomy and planetary communities in Japan, and regarded as one of the important programs for optical and infrared astronomy. It could be either an independent mission or a joining collaboration to TPF/Darwin missions.

At present, an optical high contrast space telescope (HCST) which realizes clean and stable images is under main discussions for the JTPF architecture among JTPF WG (see Figure 1). JTPF/HCST will make full use of the SPICA bus system with an optimization to shorter wavelengths. The current plan of HCST is shown in Table 4.

Table 4. JTPF/HCST preliminary specification.

\begin{tabular}{ll}
\hline Telescope & $3.5 \mathrm{~m}$ off-axis single aperture \\
Wavelength coverage & Optical and near-IR w/ limited UV capability \\
Orbit & Sun-Earth L2 \\
Instruments & Opt-NIR coronagraph, Wide-field NIR/Opt instruments \\
\hline
\end{tabular}

Various JTPF-ralated acitivies in Japan include studies of:

- combinations of nulling interferometer and modified pupil (Nishikawa et al 2005),

- a chromatic nulling of on-axial light to detect off-axial light by means of 3D commonpath interferometer (Tavrov et al. 2002), and

- polarization differential objective spectroscopy with nulling coronagraph (Murakami et al. 2005).

\section{References}

Burrows, A., Hubbard, W. B., Lunine, J. I., \& Liebert, J. 2001, Rev. of Modern Physics 73, 719 Fukagawa, M., Hayashi, M., Tamura, M. et al. 2004, ApJ 605, L53

Fukagawa, M., Tamura, M., Hayashi, M. et al. 2005, ApJ submitted

Itoh, Y., Hayashi, M., Tamura, M. et al. 2005, ApJ 620, 984

Murakami, H. 2000, ISAS Report SP 14, 267

Murakami, N., Baba, N., Tate, Y. et al. 2005, PASP submitted

Nakagawa, T. 2000, ISAS Report SP 14, 189

Nakajima, T., Morino, J., Tsuji, T. et al. 2005, Astronomical Notes in press

Neuhauser, R, Guenther, E. W., Wuchterl, G., Mugrauer, M., Bedalov, A., Hauschildt, P. H. 2005, A\& $A$ 435, L13

Nishikawa, J., Kotani, T., Murakami, N., Baba, N., Itoh, Y., \& Tamura, M. 2005, A $6 A$ 435, 379

Sato, B. et al. 2005, ApJ in press

Tamura, M., Suto, H., Takami, H. et al. 1998, SPIE 3354, 845

Tamura, M., Suto, H., Itoh, Y. et al. 2000, SPIE 4008, 1153

Tamura, M., Fukagawa, M., Kimura, H. et al. 2005, ApJ submitted

Tamura, M. \& Sato, S. 1989, AJ 98, 1368

Tavrov, A. et al. 2002, Opt. Lett. 27, 2070 